The Local Health Board of Chiavari (ASL4) received 1525 applications ( $59 \%$ on-line).

Conclusions This method has led to an increase in appropriate prescriptions and to better collaboration among medical staff.

No conflict of interest.

\section{DGI-013 ANTIRETROVIRAL TREATMENT SWITCHING IN VIROLOGICALLY UNSUPPRESSED HIV-INFECTED PATIENTS}

doi:10.1136/ejhpharm-2013-000276.279

${ }^{1} \mathrm{H}$ Navarro Aznárez, ${ }^{2} \mathrm{~A}$ Martinez-Sapiña, ${ }^{3 P}$ Arazo, 'Y Alonso, 'I Larrodé, 'N De La Llama, 'R Huarte. 'Miguel Servet Hospital, Pharmacy, Zaragoza, Spain; ${ }^{2}$ Miguel Servet Hospital, Microbiology, Zaragoza, Spain; ${ }^{3}$ Miguel Servet Hospital, Infectious Diseases, Zaragoza, Spain

Background Antiretroviral treatment (ART) has markedly decreased the morbidity and mortality due to HIV; however, in a percentage of patients a change of treatment is needed.

Purpose To determine the rates of treatment switching in HIV virologically unsuppressed patients, the reasons for changing treatment, to estimate adherence levels and to find the profiles of drugresistant mutations.

Materials and Methods Retrospective study involving patients switching ART with HIV RNA values >20 copies/ml in 2011 . Patients under 18 and those who had been on their first-line treatment no longer than 24 weeks, were excluded. Data collected: gender, age, ART and HIV RNA values before and after switching, cause of changing, adherence level (dispensing records for the last three months) and resistance testing. Data source: medical records and pharmacy database.

Results Of 1103 patients receiving ART, a total of 16\% (177) of regimens were switched, 102 cases met the inclusion criteria $(57.6 \%), 62 \%$ males, average age $44 \pm 9.5$ years. In patients switching treatment, viral load was $<500$ copies $/ \mathrm{ml}$ in $57.8 \%(59 / 102)$ $(<200$ in 51 of them $(84 \%))$. Drug-resistant mutations were assessed in $40.2 \%(41 / 102)$, and mutations were found in $41.5 \%$ of them, the more frequent mutations were: M184V (6/17), K103N (6/17), Y181C (5/17) and K65R (3/17). The main reasons for switching treatment were toxicity (52.9\%) and treatment failure (29.3\%), other reasons were regimen simplification, drug interactions and pregnancy $(17.7 \%)$. The average adherence level was $70.4 \%$, but only $38.4 \%$ of patients had high levels of adherence (>95\%). The rate of adherent patients ( $>95 \%$ ) was $55.9 \%$ in patients with viral load $<500$ copies $/ \mathrm{ml}$ versus $14.1 \%$ with viral load $>500$ ( $p<0.05)$. Conclusions Toxicity was the main reason for changing ART. The percentage of 'well-adherent' patients was very low in virologically unsuppressed HIV-infected patients, especially in those patients with high viral loads; therefore adequate adherence to treatment is a key factor in viral suppression.

No conflict of interest.

\section{DGI-014 APPROPRIATENESS OF TREATMENT AND COST ANALYSIS IN THE TREATMENT OF SYSTEMIC FUNGAL INFECTIONS IN A TRANSPLANT CENTRE}

doi:10.1136/ejhpharm-2013-000276.280

I Casucci, A Provenzani, P Polidori. Ismett, Clinical Pharmacy, Palermo, Italy

Background Invasive fungal infections (IFIs) increase morbidity and mortality in immunocompromised patients (IPs).Controlling antifungal use is fundamental in avoiding drug resistance and containing costs.

Purpose To identify risk factors associated with IFIs in IPs, and monitor appropriateness and cost of antifungal treatment.
Materials and Methods A retrospective analysis was done at ISMETT, a 78-bed transplant centre in Palermo, Italy, from 1 January to 31 December 2010. One hundred and one IPs received intravenous antifungal treatment with fluconazole $(\mathrm{F})$, liposomal amphotericin-B (A), caspofungin (C), itraconazole (I) for 4 or more days. Patient treatment was divided into three groups: prophylactic, empirical and target. Immunosuppressive treatment (IT), total parenteral nutrition (TPN), dialysis, central line, steroid treatment, stent use, neutropenia, and mechanical ventilation were evaluated. Variables were treatment duration, DDD (defined-daily-dose) consumption and DDD average cost.

Results Main risk factors were central line (65.3\%), TPN (56.4\%), dialysis $(46.5 \%)$, IT (42.6\%), mechanical ventilation (32.7\%), neutropenia (24.8\%), steroid treatment (23.8\%), and stent use $(14.9 \%)$. Average duration of prophylactic treatment was 7 days, F (61\%), A $(26 \%)$, C (13\%) were used. Average duration of empirical treatment was 8 days, and F (52.9\%), A (26.5\%), C (8.8\%), I (2.9\%), and in association $\mathrm{A}+\mathrm{C}, \mathrm{A}+\mathrm{F}, \mathrm{C}+\mathrm{F}(8.9 \%)$ were used. Average duration of target treatment was 9 days, and $\mathrm{F}(40.4 \%)$, A $(23.1 \%)$, C (15.4\%), I (7.7\%), and in association A+C, A+F, C+F (13.4\%) were used. DDD consumption and DDD average cost were, respectively, C $50 \mathrm{mg}$ vial: $273 \mathrm{DDD}$, €381.1; C $70 \mathrm{mg}$ vial: $33.6 \mathrm{DDD}$, $€ 389.6$; F $200 \mathrm{mg}$ vial: 768 DDD, €11.8; F $100 \mathrm{mg}$ vial: $89 \mathrm{DDD}$, $€ 10.6$; I $250 \mathrm{mg}$ vial: $62.5 \mathrm{DDD}, € 68.8$; and A $50 \mathrm{mg}$ vial: 2200 DDD, €93.4.

Conclusions Data showed appropriate use of antifungals. The best treatment alternative (cheaper antifungal) was prescribed for most patients. The high cost of $\mathrm{A}$ and $\mathrm{C}$ was justified by resolution of the IFI.

No conflict of interest.

\section{DGI-015 ASSESSMENT OF THE TREATMENT WITH A TWO-DRUG ANTIRETROVIRAL REGIMEN}

doi:10.1136/ejhpharm-2013-000276.281

'E Rios-Sanchez, 'MA Blanco-Castaño, 'MJ Gándara-Ladrondeguevara, 'JF LopezVallejo, ' 'JM Borrero Rubio, 'EJ Alegre-Delrey, ${ }^{2} \mathrm{MA}$ Marin-Marin. ${ }^{1}$ Hospital Universitario Puerto Real, Pharmacy, Puerto Real (Cádiz), Spain; ${ }^{2}$ Hospital Universitario Puerto Real, Infectious Diseases, Puerto Real (Cádiz), Spain

Background Antiretroviral treatment with a three drug-regimen is the initial treatment recommended for chronic HIV infection. For various reasons, the combination of three drugs can be modified to a two-drug regimen.

Purpose To analyse the change from a three-drug antiretroviral treatment regimen (HAART) to a two-drug regimen in $\mathrm{HIV}+$ patients: reason for change and effectiveness.

Materials and Methods Cross-sectional retrospective study of HIV-infected patients in treatment with two active antiretroviral drugs from January 2010 to April 2012. The data was obtained from the medical history and the Farmatools application for external patients. Effectiveness was evaluated by the viral plasma load (VPL) and the CD4 cell count, measured at 24 weeks. Viral load suppression (VLS) was defined as less than 50 copies $/ \mathrm{ml}$.

Results A total of 30 patients were studied, with the following two-drug regimens: 5 patients with boosted Atazanavir (ATZr)/ Maraviroc (MRV); 4 patients with boosted Darunavir (DRVr)/Etravirina (ETV); 13 patients with DRVr/MRV; 6 patients with DRVr/ Raltegravir (RAL); 1 patient RAL/MRV and 1 patient with boosted Fosamprenavir (FPVr)/RAL. The reasons for the change to a twodrug regimen were the following: 12 changes were determined by drug resistance tests, 6 due to side effects of previous HAART treatment and 12 to simplify their antiretroviral treatment. The answers obtained are shown in table 1 . Patients who did not reach viral load suppression at 24 weeks were taking a regimen composed of ATZr/ MRV (2 patients) and DRVr/MRV (1 patient). 